\title{
BASE-FREE FORMULAS IN THE LATTICE-THEORETIC STUDY OF COMPACTA
}

\author{
PAUL BANKSTON
}

\begin{abstract}
The languages of finitary and infinitary logic over the alphabet of bounded lattices have proven to be of considerable use in the study of compacta. Significant among the sentences of these languages are the ones that are base free, those whose truth is unchanged when we move among the lattice bases of a compactum. In this paper we define syntactically the $e x$ pansive sentences, and show each of them to be base free. We also show that many well-known properties of compacta may be expressed using expansive sentences; and that any property so expressible is closed under inverse limits and co-existential images. As a byproduct, we conclude that co-existential images of pseudo-arcs are pseudo-arcs. This is of interest because the corresponding statement for confluent maps is still open, and co-existential maps are often-but not always-confluent.
\end{abstract}

\section{INTRODUCTION}

This paper is concerned with applying the model theory of distributive lattices, along the lines of [12], to the study of how topological properties of compacta - and especially continua - are explicitly defined. (Here the word compactum refers to a compact Hausdorff space; and a continuum is a compactum that is also connected.)

Many textbook definitions of topological properties are naturally formulated in lattice-theoretic terms involving open sets and closed sets. It is our aim here to show that when these formulations have a certain well-defined syntactic shapeand there are numerous examples of these - the corresponding topological properties enjoy some nice preservation features. In particular, they are closed under images of co-existential mappings, as well as the taking of limits of inverse systems with surjective bonding maps. (We abbreviate these two closure principles respectively as "co-existential images" and "inverse limits" in the sequel.)

Because closed subsets of compacta are themselves compacta when regarded as subspaces, and because any lattice-theoretic mention of both open sets and closed sets is clearly equivalent to one involving closed sets alone, we let $F(X)=$ $\langle F(X) ; \cup, \cap, \emptyset, X\rangle$ denote the bounded lattice of all closed subsets of a topological space $X$ and confine our attention to closed-set formulations of topological properties.

From a model-theoretic perspective, $F(X)$ is an $\mathrm{L}$-structure, where $\mathrm{L}=\{\sqcup, \sqcap, \perp, \top\}$ is the alphabet for bounded lattices. By a lattice base for $X$, we mean a sublattice $\mathcal{A}$ of $F(X)$ which is also a closed-set base for $X$.

2000 Mathematics Subject Classification. Primary 03C98, 54D30; Secondary 03C20, 03C75, 03G10, 54B25, 54C10, 54F15.

Key words and phrases. compacta, continua, normal disjunctive lattices, base-free formulas, co-existential maps, inverse limits, expansive sentences. 
The reason the compact Hausdorff setting is so attractive from a model-theoretic viewpoint is that one may characterize, using simple first-order sentences, those features of an L-structure that make it isomorphic to a lattice base for a compactum. This follows easily from the work of H. Wallman [1, 18], and amounts to the following representation theorem.

Theorem 1.1. An L-structure $\mathbf{A}$ is isomorphic to a lattice base for a compactum if and only if the following three conditions hold:

(i) A satisfies the first-order axioms defining the distributive bounded lattices. (All are universal $(\forall)$ sentences.)

(ii) A satisfies the disjunctive condition; i.e., given two distinct $a, b \in \mathbf{A}$, there is some $c \neq \perp$ in $\mathbf{A}$ such that either $c \leq a$ and $c \sqcap b=\perp$ or $c \leq b$ and $c \sqcap a=\perp$. (This is expressible using a universal-existential $(\forall \exists$ ) sentence, where $a \leq b$ abbreviates $a \sqcap b=a$.)

(iii) A satisfies the normality condition; i.e., given $a, b \in \mathbf{A}$ with $a \sqcap b=\perp$, there are $a^{\prime}, b^{\prime} \in \mathbf{A}$ such that $a \sqcap a^{\prime}=b \sqcap b^{\prime}=\perp$ and $a^{\prime} \sqcup b^{\prime}=\top$. (This is also expressible using $a \forall \exists$ sentence.)

An L-structure is a normal disjunctive lattice if it satisfies the first-order conditions above. A lattice base for a compactum is then a normal disjunctive lattice; conversely, if $\mathbf{A}$ is a normal disjunctive lattice, there are a canonically-defined compactum $X=S(\mathbf{A})$ and a lattice base $\mathcal{A}$ for $X$ such that $\mathbf{A}$ and $\mathcal{A}$ are isomorphic lattices. The points of $X$ are the maximal filters of $\mathbf{A}$; the elements of $\mathcal{A}$ are the sets $a^{\sharp}:=\{\mu \in X: a \in \mu\}$, for $a \in \mathbf{A}$. The assignment $a \mapsto a^{\sharp}$ defines an isomorphism; the reader will recognize $X$ is the classic maximal spectrum - also known as the Wallman representation - for the lattice $\mathbf{A}$.

In this paper we consider topological properties definable using sentences from the infinitary language $\mathrm{L}_{\omega_{1} \omega}$ (see, e.g., [13]), where the only syntactic formation rule beyond the usual first-order ones is infinitary disjunction $\bigvee \Phi$ applied to a countable set of formulas, the union of whose sets of free variables is finite. If $\Delta$ is a set of sentences in this language, then the property $\mathfrak{P}_{\Delta}$ defined by $\Delta$ is the class of compacta whose closed-set lattices satisfy all sentences in $\Delta$.

Two different lattice bases for a single compactum $X$ may easily fail to satisfy even the same finitary (i.e., first-order) sentences; indeed $F(X)$ is always atomic, but a lattice base for $X$ may well be atomless. So we define an $\mathrm{L}_{\omega_{1} \omega}$-formula $\varphi\left(x_{0}, \ldots, x_{n-1}\right)$ (with distinct free variables among $\left\{x_{0}, \ldots, x_{n-1}\right\}$ ) to be base free if whenever $X$ is a compactum, $\mathcal{A}$ is a lattice base for $X$ and $\left\langle A_{0}, \ldots, A_{n-1}\right\rangle \in \mathcal{A}^{n}$, we have $\mathcal{A} \models \varphi\left[A_{0}, \ldots, A_{n-1}\right]$ if and only if $F(X) \models \varphi\left[A_{0}, \ldots, A_{n-1}\right]$. (Here the notation indicates the substitution of constant $A_{i}$ for the variable $x_{i}, i<n$, in the standard way. We often let $\bar{x}$ abbreviate the variable string $\left\langle x_{0}, \ldots, x_{n-1}\right\rangle$ when we do not need to be specific about its length.) So when $\Delta$ is a set of base-free sentences, the class of normal disjunctive lattices $\mathbf{A}$ with $S(\mathbf{A}) \in \mathfrak{P}_{\Delta}$ is defined by $\Delta$, plus the first-order conditions in Theorem 1.1 .

Remark 1.2. It is easy to show that a Boolean combination - possibly infinitaryof base-free formulas is base free; the problem is with the introduction of quantification. As a simple example, consider the formula $\varphi(x)$, defined to be 
$[x \neq \perp \wedge \forall y(y \leq x \rightarrow(y=\perp \vee y=x))]$, that says of a lattice element that it is an atom. This formula is base free, although its existential closure $\exists x \varphi(x)$ is not.

Recall that a $\forall \bigvee \exists$ formula of $\mathrm{L}_{\omega_{1} \omega}$ is a formula $\varphi$ with the syntactic shape $\forall \bar{x} \bigvee \Psi$, where each formula in $\Psi$ is of the form $\exists \bar{y} \psi$, such that $\psi$ is quantifier free. The formulas $\exists \bar{y} \psi$ are called the constituents of $\varphi$. We say $\varphi$ is constituent base free if each of its constituents is base free.

In the sequel we show that if a property $\mathfrak{P}$ of compacta is definable by a set $\Delta$ of $\forall \bigvee \exists$ sentences that are base free, then $\mathfrak{P}$ is closed under inverse limits. If the sentences in $\Delta$ are constituent base free, then $\mathfrak{P}$ is closed under co-existential images. These preservation results are actually quite straightforward, but are important for setting up the next step; which is to describe syntactically the set of $\forall \bigvee \exists$ sentences of $\mathrm{L}_{\omega_{1} \omega}$ that are expansive, and to show that each expansive sentence is both base free and constituent base free. Finally we give a list of important compactum-theoretic properties that may (may not) be specified using expansive sentences.

\section{CLOSURE UNDER INVERSE LIMITS}

The inverse limit construction is one of the most basic in topology, especially in continuum theory (see, e.g., [17]). For simplicity, we choose the natural numbers $\omega:=\{0,1, \ldots\}$ for our directed index set, but this is not an essential restriction.

Recall that if $X_{0} \stackrel{f_{0}}{\leftarrow} X_{1} \stackrel{f_{1}}{\leftarrow} \ldots$ is a sequence of spaces and mappings, the inverse limit is the subspace of the usual cartesian product $\prod_{n<\omega} X_{n}$ whose elements are sequences $\left\langle x_{0}, x_{1}, \ldots\right\rangle$ such that $x_{n}=f_{n}\left(x_{n+1}\right), n<\omega . X_{n}$ and $f_{n}$ are referred to as the $n$th coordinate space and $n$th bonding map, respectively. We confine our attention to inverse systems whose coordinate spaces are compacta and whose bonding maps are surjective. Then the inverse limits are nonempty when the coordinate spaces are; moreover, inverse limits of continua are continua.

Proposition 2.1. Let $\Delta$ be a set of $\forall \bigvee \exists$ sentences that are base free. If $X_{0} \stackrel{f_{0}}{\leftarrow}$ $X_{1} \stackrel{f_{1}}{\leftarrow} \ldots$ is an inverse system and $X_{n} \in \mathfrak{P}_{\Delta}$ for each $n<\omega$, then the inverse limit is in $\mathfrak{P}_{\Delta}$ also.

Proof. For each $n<\omega$, let $f_{n}^{F}: F\left(X_{n}\right) \rightarrow F\left(X_{n+1}\right)$ be the lattice homomorphism defined by taking full pre-images. Then, because each $f_{n}$ is surjective, we have a direct system of normal disjunctive lattices and embeddings. Denote the direct limit structure by $\mathbf{A}$. We then use Theorem 1.1, together with (the easy direction of ) the Chang-Łoś-Suszko theorem, which applies to $\forall \bigvee \exists$ sentences just as well as $\forall \exists$ ones (see [8]), to infer that $\mathbf{A}$ is a normal disjunctive lattice that satisfies the sentences in $\Delta$. And since these sentences are base free, they are also true in $F(S(\mathbf{A})$ ), which is easily shown to be isomorphic to the closed set lattice for the inverse limit. 


\section{CLOSURE UNDER CO-EXISTENTIAL IMAGES}

Co-existential maps are defined using topological ultracopowers in an exact mirroring of how one characterizes the existential embeddings of model theory using ultrapowers. (See, e.g., [3] for a full explanation.) What is more, if $f: \mathbf{A} \rightarrow \mathbf{B}$ is any existential embedding between normal disjunctive lattices, then the $S$-induced mapping $f^{S}: S(\mathbf{B}) \rightarrow S(\mathbf{A})$ - defined by taking $f^{S}(\mu)$ to be the unique maximal filter in A that contains the pre-image $f^{-1}[\mu]$ (itself a prime filter) - is automatically a co-existential map between compacta.

The formal definition of when a mapping $f: X \rightarrow Y$ between compacta is coexistential is that there is some ultracopower $Y_{\mathcal{D}}$, with canonical projection map $p_{Y, \mathcal{D}}: Y_{\mathcal{D}} \rightarrow Y$, and a continuous surjection $g: Y_{\mathcal{D}} \rightarrow X$ such that the following diagram commutes:

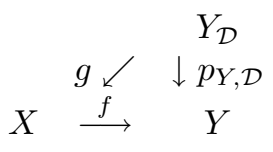

To elaborate, ultracopowers are special cases of ultracoproducts when all the factor spaces are the same. Assuming the reader familiar with the usual ultraproduct construction, we know from Theorem 1.1, plus the Fundamental Theorem of Ultraproducts of J. Łoś (see [8]), that ultraproducts of normal disjunctive lattices are normal disjunctive lattices. So if $\left\langle X_{i}: i \in I\right\rangle$ is an $I$-indexed family of compacta, with $\mathcal{D}$ an ultrafilter on $I$, we may define the $\mathcal{D}$-ultracoproduct $\sum_{\mathcal{D}} X_{i}$ of this family to be the Wallman representation $S\left(\prod_{\mathcal{D}} F\left(X_{i}\right)\right)$. This construction is stable in the following sense: Suppose $\mathcal{A}_{i}$ is a lattice base for $X_{i}, i \in I$, with $f_{i}: \mathcal{A}_{i} \rightarrow F\left(X_{i}\right)$ the corresponding inclusion homomorphism. Let $f: \prod_{\mathcal{D}} \mathcal{A}_{i} \rightarrow \prod_{\mathcal{D}} F\left(X_{i}\right)$ be the usual ultraproduct homomorphism, with $f^{S}: S\left(\prod_{\mathcal{D}} F\left(X_{i}\right)\right) \rightarrow S\left(\prod_{\mathcal{D}} \mathcal{A}_{i}\right)$ its $S$-induced mapping. Then $f^{S}$ is a homeomorphism [3].

When the spaces $X_{i}$ are all the same space $X$, the ultracopower is abbreviated $X_{\mathcal{D}}$. In this case there is a canonical projection map $p=p_{X, \mathcal{D}}: X_{\mathcal{D}} \rightarrow X$, defined by the condition $p(\mu)=x$ if and only if the $\mathcal{D}$-ultrapower of the closure of every open neighborhood of $x$ is a member of $\mu$. This completes the definition of what it means for a mapping between compacta to be co-existential.

Co-existential mappings pair up with inverse limits using surjective bonding maps on the topological side much as existential embeddings pair up with direct limits of embeddings on the model-theoretic side. In fact, if $\mathfrak{P}$ is a property of compacta that is both closed under the ultracoproduct construction and the condition that compactum $X$ has property $\mathfrak{P}$ whenever some ultracopower of $X$ has the property, then $\mathfrak{P}$ is closed under co-existential images if and only if it is closed under inverse limits. This is the Chang-Łoś-Suszko theorem in a topological setting [4]; in our situation, however, we travel outside the first-order perimeter to consider properties - e.g., chainability - that are defined using essentially infinitary language. 
Remark 3.1. Co-existential mappings are special, but not particularly rare. Indeed, every mapping from a compactum onto a zero-dimensional compactum without isolated points is co-existential. Moreover, every compactum (resp., continuum) is a continuous image of a compactum (resp., continuum) that is itself only a coexistential image of other compacta (resp., continua). (See [3] for details.) Finally [7], a compactum is locally connected if and only if every co-existential mapping onto it is monotone (i.e., pre-images of subcontinua are subcontinua).

Proposition 3.2. Let $\Delta$ be a set of $\forall \bigvee \exists$ sentences that are constituent base free. If $X \in \mathfrak{P}_{\Delta}$ and $f: X \rightarrow Y$ is a co-existential map, then $Y \in \mathfrak{P}_{\Delta}$ also.

Proof. Let $g: Y_{\mathcal{D}} \rightarrow X$ be a continuous surjection witnessing the co-existentiality of $f: X \rightarrow Y$, with $p=p_{Y, \mathcal{D}}$. As in the proof of Proposition 2.1, we apply the contravariant functor $F$ to this commutative triangle, obtaining a new commutative triangle of closed-set lattices and lattice embeddings. The $\mathcal{D}$-ultrapower $F(Y)^{\mathcal{D}}$ of $F(Y)$ is then the standard lattice base for the $\mathcal{D}$-ultracopower $Y_{\mathcal{D}}$. Let $d: F(Y) \rightarrow$ $F(Y)^{\mathcal{D}}$ be the ultrapower diagonal embedding, with $e: F(Y)^{\mathcal{D}} \rightarrow F\left(Y_{\mathcal{D}}\right)$ the inclusion homomorphism. Then $p^{F}=e \circ d$, and we have a commutative square of normal disjunctive lattices, with the embedding $p^{F}$ forming a diagonal for the square.

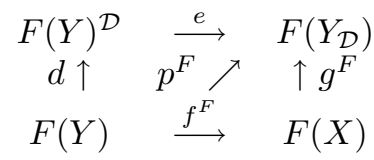

Let $\Delta$ be as in the statement of the proposition, and suppose $F(X) \models \Delta$. Pick $\delta \in \Delta$; for simplicity, and without loss of generality, we may assume $\delta$ is of the form $\forall x \exists y \psi(x, y)$. We wish to prove $F(Y) \models \delta$, so let $A \in F(Y)$ be an arbitrary closed subset of $Y$. Then, by hypothesis, there is some $B \in F(X)$ such that $F(X) \models \psi\left[f^{F}(A), B\right]$. Since $\psi(x, y)$ is quantifier free, we know that $F\left(Y_{\mathcal{D}}\right) \models$ $\psi\left[p^{F}(A), g^{F}(B)\right]$; hence $F\left(Y_{\mathcal{D}}\right) \models \exists y \psi\left[p^{F}(A), y\right]$. Now $p^{F}(A)=e(d(A))$ and $\exists y \psi$ is base free; so $F(Y)^{\mathcal{D}} \models \exists y \psi[d(A), y]$. Since $d$ is an elementary embedding, we immediately have $F(Y) \models \exists y \psi[A, y]$. Thus $F(Y) \models \delta$, and we infer that $Y \in \mathfrak{P}_{\Delta}$.

\section{EXPANSIVE SENTENCES}

Officially all atomic formulas of $\mathbf{L}_{\omega_{1} \omega}$ are equations of the form $\sigma=\tau$, where $\sigma$ and $\tau$ are lattice-theoretic terms. A formula is called restricted if each of its atomic subformulas is an equation with at least one side $\perp$ or $T$. As above, we often use $\sigma \leq \tau$ to abbreviate either of the two nonrestricted formulas $\sigma \sqcap \tau=\sigma$ or $\sigma \sqcup \tau=\tau$ (being equivalent to one another in any lattice). For convenience we partition our set of variables into $\left\{x_{0}, x_{1}, \ldots\right\}$ and $\left\{y_{0}, y_{1}, \ldots\right\}$. We call a formula $\eta(\bar{x}, \bar{y})$ weakly linking if it is a positive Boolean combination (i.e., no negations) of formulas of the following three types: 
(1) $\sigma(\bar{x}) \leq \tau(\bar{y})$

(2) $\neg[\tau(\bar{y}) \leq \sigma(\bar{x})]$

(3) quantifier-free restricted.

We define a weakly linking formula to be linking if the formulas allowed in (3)

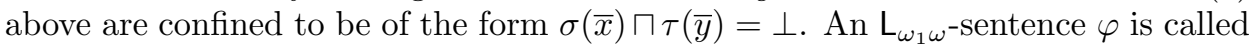
weakly expansive (resp., expansive) if it is of the form

$$
\forall \bar{x}[\alpha(\bar{x}) \rightarrow \bigvee \Psi(\bar{x})]
$$

where:

- $\alpha(\bar{x})$ is restricted and quantifier free; and

- each formula of $\Psi(\bar{x})$ is of the form $\exists \bar{y}(\eta(\bar{x}, \bar{y}) \wedge \psi(\bar{y}))$, where $\eta(\bar{x}, \bar{y})$ is a weakly linking (resp., linking) formula, and $\psi(\bar{y})$ is restricted and quantifier free.

Clearly a weakly expansive sentence may be written in $\forall \bigvee \exists$ format, and its constituent formulas may be taken to be of the form

$$
\alpha(\bar{x}) \rightarrow \exists \bar{y}(\eta(\bar{x}, \bar{y}) \wedge \psi(\bar{y}))
$$

Our objective in this section is to show that expansive sentences are both base free and constituent base free. To this end, let us make the following definition. For two $m$-tuples $\left\langle a_{0}, \ldots, a_{m-1}\right\rangle,\left\langle b_{0}, \ldots, b_{m-1}\right\rangle$ from a lattice $\mathbf{A}$, we say the second is an exact corefinement of the first if: (i) $b_{j} \geq a_{j}, j<m$; and (ii) for each restricted quantifier-free formula $\rho\left(y_{0}, \ldots, y_{m-1}\right)$ over $\mathbf{L}$, we have $\mathbf{A}=\rho\left[a_{0}, \ldots, a_{m-1}\right]$ if and only if $\mathbf{A}=\rho\left[b_{0}, \ldots, b_{m-1}\right]$. This sharpens the idea of a swelling, introduced in the 1930s for studying covering dimension. The following lemma is a strengthened version of Theorem 7.1.4 in [10], the principal result concerning swellings.

Lemma 4.1. Let $X$ be a compactum, with $\mathcal{B}$ a lattice base for $X$. Then every $m$-tuple of sets from $F(X)$ has an exact corefinement consisting of sets from $\mathcal{B}$.

Proof. We first observe that terms $\sigma\left(y_{0}, \ldots, y_{m-1}\right)$ over $\mathrm{L}$ are monotonic, in the sense that if $\mathbf{A}$ is any lattice, with $a_{j} \leq b_{j}, j<m$, then $\mathbf{A} \models\left(\sigma\left[a_{0}, \ldots, a_{m-1}\right] \leq\right.$ $\left.\sigma\left[b_{0}, \ldots, b_{m-1}\right]\right)$. From this we conclude that if $\rho\left(y_{0}, \ldots, y_{m-1}\right)$ is a restricted atomic formula, $A_{j} \subseteq B_{j} \subseteq C_{j}, j<m$, are sets from $F(X)$, and $F(X) \models\left(\rho\left[A_{0}, \ldots, A_{m-1}\right] \wedge \rho\left[C_{0}, \ldots, C_{m-1}\right]\right)$ 
(resp., $\left.F(X) \models\left(\neg \rho\left[A_{0}, \ldots, A_{m-1}\right] \wedge \neg \rho\left[C_{0}, \ldots, C_{m-1}\right]\right)\right)$, then $F(X) \models \rho\left[B_{0}, \ldots, B_{m-1}\right]$ (resp., $\left.F(X) \models \neg \rho\left[B_{0}, \ldots, B_{m-1}\right]\right)$.

We next observe that it suffices to establish the stated result for restricted formulas that are either atomic or negated atomic, as restricted quantifier-free formulas are just positive Boolean combinations of these.

Let $\left\langle A_{0}, \ldots, A_{m-1}\right\rangle$ be a fixed $m$-tuple from $F(X)$. For each restricted atomic (or negated atomic) formula $\rho\left(y_{0}, \ldots, y_{m-1}\right)$, we construct an $m$-tuple $\left\langle B_{0}^{\rho}, \ldots, B_{m-1}^{\rho}\right\rangle$ from $\mathcal{B}$ in a straightforward way, à la the proof of Theorem 7.1.4 in [10]. In particular, we have $A_{j} \subseteq B_{j}$, for $j<m$; and $F(X) \models \rho\left[A_{0}, \ldots, A_{m-1}\right]$ if and only if $F(X) \models \rho\left[B_{0}, \ldots, B_{m-1}\right]$. The proof naturally splits into the cases where one side of $\rho$ is $\perp$ or $T$, and we repeatedly use the observation in the first paragraph. Finally, we note that $\rho$ may be taken to range over a finite set $\left\{\rho_{0}, \ldots, \rho_{r-1}\right\}$ of formulas in the variables $y_{0}, \ldots, y_{m-1}$. With this in mind, we set $B_{j}:=B_{j}^{\rho_{0}} \cap \cdots \cap B_{j}^{\rho_{r-1}}$, for each $j<m$. Then $\left\langle B_{0}, \ldots, B_{m-1}\right\rangle$ is an exact corefinement of $\left(A_{0}, \ldots, A_{m-1}\right)$, consisting of sets from $\mathcal{B}$.

The main result connecting syntax with semantics is now the following.

Theorem 4.2. Weakly expansive sentences are constituent base free; expansive sentences are also base free.

Proof. Let $\varphi$ be the weakly expansive sentence $\forall \bar{x}[\alpha(\bar{x}) \rightarrow \bigvee \Psi(\bar{x})]$. To show $\varphi$ is constituent base free, let $\gamma(\bar{x})$ be one of its constituent formulas $\alpha(\bar{x}) \rightarrow \exists \bar{y}(\eta(\bar{x}, \bar{y}) \wedge$ $\psi(\bar{y})$ ), where $\bar{x}=\left\langle x_{0}, \ldots, x_{m-1}\right\rangle$ and $\bar{y}=\left\langle y_{0}, \ldots, y_{n-1}\right\rangle$. Let $X$ be a compactum with lattice base $\mathcal{A}$. Given $\bar{A}=\left\langle A_{0}, \ldots, A_{m-1}\right\rangle \in \mathcal{A}^{m}$, it suffices to show that $\mathcal{A} \models \gamma[\bar{A}]$ if $F(X) \models \gamma[\bar{A}]$. (The other implication is, of course, trivial.)

So suppose $\mathcal{A} \models \alpha[\bar{A}]$. Then $F(X) \models \alpha[\bar{A}]$; hence there is some $\bar{B}=\left\langle B_{0}, \ldots, B_{n-1}\right\rangle \in F(X)^{n}$ such that $F(X) \models(\eta[\bar{A}, \bar{B}] \wedge \psi[\bar{B}])$. Using Lemma 4.1 , we get an exact corefinement

$\left\langle A_{0}^{\prime}, \ldots, A_{m-1}^{\prime}, B_{0}^{\prime}, \ldots, B_{n-1}^{\prime}\right\rangle$ of $\left\langle A_{0}, \ldots, A_{m-1}, B_{0}, \ldots, B_{n-1}\right\rangle$ consisting of sets from $\mathcal{A}$. It is easy to show that if $A_{i} \subseteq A_{i}^{\prime \prime} \subseteq A_{i}^{\prime}$, for $i<m$, and $B_{j} \subseteq B_{j}^{\prime \prime} \subseteq B_{j}^{\prime}$, for $j<n$, then $\left\langle A_{0}^{\prime \prime}, \ldots, A_{m-1}^{\prime \prime}, B_{0}^{\prime \prime}, \ldots, B_{n-1}\right\rangle$ is also an exact corefinement of $\left\langle A_{0}, \ldots, A_{m-1}, B_{0}, \ldots, B_{n-1}\right\rangle$. In particular, $\left\langle A_{0}, \ldots, A_{m-1}, B_{0}^{\prime}, \ldots, B_{n-1}^{\prime}\right\rangle$ is an exact corefinement of $\left\langle A_{0}, \ldots, A_{m-1}, B_{0}, \ldots, B_{n-1}\right\rangle$. Let $\bar{B}^{\prime}=\left\langle B_{0}^{\prime}, \ldots, B_{n-1}^{\prime}\right\rangle$. Then it is also true that $\bar{B}^{\prime}$ is an exact corefinement of $\bar{B}$; and, since $\psi(\bar{y})$ is restricted and quantifier free, we know that $F(X) \models \psi\left[\bar{B}^{\prime}\right]$. Therefore $\mathcal{A} \models \psi\left[\bar{B}^{\prime}\right]$; so it remains to show that $\mathcal{A} \models \eta\left[\bar{A}, \bar{B}^{\prime}\right]$.

Now since terms over $\mathrm{L}$ are monotonic (see the proof of Lemma 4.1 ), we know that if $\eta(\bar{x}, \bar{y})$ is of either of the first two types given above and $F(X) \models \eta[\bar{A}, \bar{B}]$, then $F(X) \models \eta\left[\bar{A}, \bar{B}^{\prime}\right]$; hence $\mathcal{A} \models \eta\left[\bar{A}, \bar{B}^{\prime}\right]$. If $\eta(\bar{x}, \bar{y})$ is of the third type, restricted and quantifier free, we may directly apply the definition of exact corefinement. Since $\eta(\bar{x}, \bar{y})$ in general is just a positive Boolean combination of formulas of these three types, we may now infer that $\mathcal{A} \models \eta\left[\bar{A}, \bar{B}^{\prime}\right]$, as desired. This is just what we need to show $\mathcal{A} \models \gamma[\bar{A}]$, and hence that the weakly expansive sentence $\varphi$ is constituent base free. 
To show $\varphi$ is base free when it is expansive, there are two implications to establish. If $F(X) \models \varphi$ and $\bar{A} \in \mathcal{A}^{m}$, then $F(X) \models \gamma[\bar{A}]$ for some constituent formula $\gamma(\bar{x})$ of $\varphi$. By the argument above, we have $\mathcal{A} \models \gamma[\bar{A}]$; and so $\mathcal{A} \models \varphi$. Now suppose $\mathcal{A} \models \varphi$. Assume $\bar{A}=\left\langle A_{0}, \ldots, A_{m-1}\right\rangle \in F(X)^{m}$ is such that $F(X) \models \alpha[\bar{A}]$. Then, using Lemma 4.1, there is an exact corefinement $\bar{A}^{\prime}=\left\langle A_{0}^{\prime}, \ldots, A_{m-1}^{\prime}\right\rangle \in \mathcal{A}^{m}$ of $\bar{A}$; so we infer $\mathcal{A} \models \alpha\left(\bar{A}^{\prime}\right)$. Hence there is some $\exists \bar{y}(\eta(\bar{x}, \bar{y}) \wedge \psi(\bar{y}))$ in $\Psi(\bar{x})$ and some $\bar{B} \in \mathcal{A}^{n}$ such that $\mathcal{A} \models\left(\eta\left[\bar{A}^{\prime}, \bar{B}\right] \wedge \psi[\bar{B}]\right)$. Thus $F(X) \models\left(\eta\left[\bar{A}^{\prime}, \bar{B}\right] \wedge \psi[\bar{B}]\right)$, and it remains to show that $F(X) \models \eta[\bar{A}, \bar{B}]$. This follows as in the last paragraph, with the one difference that we invoke monotonicity of terms also when $\eta(\bar{x}, \bar{y})$ is of the form $\sigma(\bar{x}) \sqcap \tau(\bar{y})=\perp$. (We may not simply invoke the fact that $\eta(\bar{x}, \bar{y})$ is restricted and quantifier free because $\left\langle A_{0}^{\prime}, \ldots, A_{m-1}^{\prime}, B_{0}, \ldots, B_{n-1}\right\rangle$ may not be an exact corefinement of $\left\langle A_{0}, \ldots, A_{m-1}, B_{0}, \ldots, B_{n-1}\right\rangle$.) This completes the proof that $\varphi$ is base free.

Putting Propositions 2.1 and 3.2 together with Theorem 4.2 immediately yields the following result.

Corollary 4.3. Let $\Delta$ be a set of weakly expansive (resp., expansive) sentences. Then $\mathfrak{P}_{\Delta}$ is closed under co-existential images (resp., co-existential images and inverse limits).

\section{SOME PROPERTIES THAT ARE (ARE NOT) EXPANSIVE}

We begin with two well-known properties that are not expansive.

Examples 5.1. (1) (Decomposable) This is the property of a continuum that says it is the union of two proper subcontinua. Any continuum that can be easily visualized - such as an arc (i.e., a topological copy of a closed bounded interval in the real line)-is decomposable, and while this property should apply to any nondegenerate continuum, it actually does not. Indeed it fails to be closed under either inverse limits [17] or co-existential images [3]. Hence it is not an expansive property (or even a weakly expansive one).

(2) (Locally connected) This is the property that each point has a neighborhood base of connected open sets, and is also one that applies to easily visualizable continua. And while it is closed under co-existential images - indeed, all topological quotients - it is not closed under inverse limits [17]. This tells us that local connectedness is not an expansive property, but leaves open the question of whether it is weakly expansive.

In the following group of examples, we consider how certain properties of compacta may be formulated using finitary (weakly) expansive sentences $\forall \bar{x}[\alpha(\bar{x}) \rightarrow$ $\exists \bar{y}(\eta(\bar{x}, \bar{y}) \wedge \psi(\bar{y})]$.

Examples 5.2. (1) (Connected) In this simple case, the $\bar{y}$ string is empty; $\alpha\left(x_{0}, x_{1}\right)$ is $\left(x_{0} \sqcap x_{1}=\perp\right) \wedge\left(x_{0} \sqcup x_{1}=\top\right)$ and $\eta\left(x_{0}, x_{1}\right)$ is $x_{0}=\top \vee x_{1}=\top$. 
[In all subsequent examples of properties of continua, we describe just the sentences we need to add to the statement of connectedness.]

(2) (Indecomposable) Indecomposability is the negation of decomposability, and says of a continuum $X$ that if $X$ is the union of two proper closed subsets $A$ and $B$, then at least one of the subsets must have a disconnection. This is easily expressible as a weakly expansive finitary sentence, but we can do better. To obtain an expansive formulation, we resort to a characterization of indecomposability given in [4] (Theorem 4.5), namely that whenever $A$ and $B$ are closed in $X$ with $A \cup B=X$ and $A \neq X \neq B$, there exist closed sets $H$ and $K$ of $X$ such that: (i) $A \subseteq H \cup K$; (ii) $H \nsubseteq B$ and $K \nsubseteq B$; and (iii) $A \cap(H \cap K)=\emptyset$.

(3) (Hereditarily indecomposable) A continuum $X$ is hereditarily indecomposable if any two subcontinua are either disjoint or comparable. The most famous hereditarily indecomposable continuum is the pseudo-arc (see, e.g., $[17,16])$. This definition is certainly not readily expressible even as a weakly expansive sentence; fortunately there is the following characterization, due to Krasinkiewicz and Minc [14], which is easily formulable as a finitary expansive sentence: Whenever $A, A^{\prime}, B, B^{\prime}$ are closed subsets, with $A$ disjoint from $B$ and $A \cap A^{\prime}=B \cap B^{\prime}=\emptyset$, then there exist closed sets $H, K$ and $M$ such that: (i) $A \subseteq H$ and $B \subseteq M$; (ii) $A^{\prime} \cap(K \cap M)=\emptyset=B^{\prime} \cap(H \cap K)$; and (iii) $H \cap M=\emptyset$ and $H \cup K \cup M=X$.

(4) (Unicoherent) A continuum $X$ is unicoherent if whenever $A$ and $B$ are two subcontinua such that $X=A \cup B$, it follows that $A \cap B$ is connected. This is a very general way of saying that there are no "holes" in the continuum. Cells of dimension $\geq 1$ and spheres of dimension $\geq 2$ are unicoherent; circles and lemniscates are not. In [5] it was proved using model theory that there must be some $\forall \exists$ base-free sentence that characterizes unicoherence, but no such sentence is currently known. Here we partially rectify the situation by providing a weakly expansive sentence expressing unicoherence. First we state a simple lemma, whose proof is an easy exercise.

Lemma 5.3. Let $D$ be a closed subset of a normal space $X$. Then $D$ is disconnected if and only if there are closed subsets $R, S \subseteq X$ such that: (i) $R \cup S=X$; (ii) $D \cap R \neq \emptyset \neq D \cap S$; and (iii) $D \cap(R \cap S)=\emptyset$.

A finitary weakly expansive formulation of unicoherence in a continuum $X$ simply says that for all closed sets $A, B, R, S$; if $A \cup B=X=R \cup S$, $(A \cap B) \cap R \neq \emptyset \neq(A \cap B) \cap S$, and $(A \cap B) \cap(R \cap S)=\emptyset$, then (for $C=A$ or for $C=B$ ) there are closed sets $H, K$ such that: (i) $C \subseteq H \cup K$; (ii) $C \cap(H \cap K)=\emptyset$; and (iii) (the only condition that does not fit the criteria for being expansive) $H \cap C \neq \emptyset \neq K \cap C$.

The next several examples constitute what are commonly referred to as "covering properties." In the $\mathrm{L}_{\omega_{1} \omega}$ sentence $\forall \bar{x}[\alpha(\bar{x}) \rightarrow \bigvee \Psi(\bar{x})], \alpha\left(x_{0}, \ldots, x_{k-1}\right)$ says that 
"the sets $x_{0}, \ldots, x_{k-1}$ form a closed co-cover," i.e., that their complements, once interpreted in some $F(X)$, form an open cover. Thus $\alpha$ is just the restricted atomic formula $x_{0} \sqcap \cdots \sqcap x_{k-1}=\perp$. Each linking formula $\eta\left(x_{0}, \ldots, x_{k-1}, y_{0}, \ldots, y_{m-1}\right)$ is the corefinement condition $\bigwedge_{j<m} \bigvee_{i<k}\left(x_{i} \leq y_{j}\right)$ (each $y_{j}$ "contains" some $x_{i}$ ); so it remains to specify the formulas $\psi(\bar{y})$.

Examples 5.4. (1) (Dimension $\leq n$ ) The covering dimension of a compactum is $\leq n, n<\omega$, if for each finite open cover there is a refining open cover, no $n+2$ of whose sets have a common intersection. So for each fixed $m=1,2, \ldots, \psi_{m}\left(y_{0}, \ldots, y_{m-1}\right)$ is the conjunction of the formulas

$$
\left(y_{0} \sqcap \cdots \sqcap y_{m-1}=\perp\right) \wedge\left(\sqcup\left\{y_{j}: j \in s\right\}=\top\right),
$$

as $s$ ranges over all subsets of $\{0, \ldots, m-1\}$, of cardinality $n+2$.

(2) (Chainable) A compactum is chainable if for each finite open cover there is a refining open cover that forms a "chain." Chainable compacta are automatically continua; the arc (resp., pseudo-arc) is the only metrizable continuum that is both locally connected (resp., hereditarily indecomposable) and chainable $[17,16]$. In this case $\psi\left(y_{0}, \ldots, y_{m-1}\right)$ is the conjunction of the formulas

$$
\begin{gathered}
y_{0} \sqcap \cdots \sqcap y_{m-1}=\perp, \\
\left\{y_{i} \sqcup y_{j} \neq \top: i, j<m,|i-j| \leq 1\right\}, \text { and } \\
\left\{y_{i} \sqcup y_{j}=\top: i, j<m,|i-j| \geq 2\right\} .
\end{gathered}
$$

(3) (Acyclic curve) A compactum is acyclic (see, e.g., [9]) if it is connected and is such that every continuous map from it into a simple closed curve (i.e., a homeomorphic copy of the standard unit circle in the complex plane) is nullhomotopic. If, in addition, it is of covering dimension 1 , it is called an acyclic curve. In [2] it is proved (Theorem 2.1) that a compactum is an acyclic curve if and only if for each three-set open cover there is a refining open cover that forms a chain in the sense used above. So this amounts to just a simpler version of (2).

(4) (G-refinable) Let $\mathcal{G}$ be a family of topological graphs; i.e., each of its members is a metrizable compactum that is decomposable into a finite union of points and arcs, no two arcs of which intersect in a cut point of either. We may assume that the members of $\mathcal{G}$ are pairwise nonhomeomorphic, and hence that $\mathcal{G}$ may be countably indexed as $\left\{G_{r}: r<\omega\right\}$. A compactum is $\mathcal{G}$-refinable if for each finite open cover there is a refining open cover whose nerve is an abstract graph that realizes topologically as a compactum homeomorphic to some $G_{r}$. Thus chainability (resp., circular chainability) is $\{G\}$-refinability, where $G$ is an arc (resp., a simple closed curve). Also, when $\mathcal{G}$ is the family of all topological trees-i.e., connected topological graphs containing no simple closed curves - the property of being $\mathcal{G}$-refinable is what many authors call being tree-like. Tree-like continua are well known [9] to be acyclic curves. It is not hard to show, using an inductive argument involving the number of arcs and points used to describe 
a topological graph, that $\mathcal{G}$-refinability is an expansive covering property.

We end with some remarks and observations.

Remarks 5.5. (1) In [2] and [5] a property $\mathfrak{P}$ is finitely expressible if there is a finitary base-free sentence over $\mathrm{L}$ such that a compactum $X$ is in $\mathfrak{P}$ just in case $F(X)$ satisfies the sentence. Thus all the properties in Example 5.2 are finitely expressible. In addition, for each $n<\omega$, the property of being of covering dimension $\leq n$ is finitely expressible, thanks to a famous theorem of E. Hemmingsen. However, chainability and the property of being an acyclic curve are not. (See $[2,4]$ for details.)

(2) Referring to Example 5.4(3), we do not know whether co-existential maps preserve acyclicity without the dimension restriction. In [15], A. Lelek proved that acyclicity is preserved by maps that are confluent, i.e., ones for which each component of the pre-image of a subcontinuum of the range is sent by that map onto the subcontinuum. This significantly improves on a 1930s result of S. Eilenberg, who showed preservation in the case the map is either monotone or open.

(3) Because both chainability and hereditary indecomposability are expansive properties, together with the fact that the pseudo-arc is unique among the chainable hereditarily indecomposable continua that are metrizable, we know that a co-existential image of a pseudo-arc is also a pseudo-arc. It is known [16] that confluent maps preserve being tree-like; however it is not currently known whether they preserve chainability. In particular, it is not known whether they preserve being a pseudo-arc. Co-existential mappings are monotone when the image space is locally connected, and are weakly confluent (i.e., some component of the pre-image of a subcontinuum maps onto the subcontinuum) in general [3]. Recently [6,11], examples have been constructed to show that co-existential mappings need not be confluent. For the converse, confluent mappings - even ones that are both monotone and open - may raise dimension [19], unlike co-existential mappings [3].

\section{REFERENCES}

[1] J. M. Aarts, Wallman-Shanin Compactification, Encyclopedia of General Topology (K. P. Hart, J. Nagata, and J. E. Vaughan, eds.) Elsevier Science Publishers B. V., Amaterdam, 2004, pp. 218-220.

[2] T. Banakh, P. Bankston, B. Raines, and W. Ruitenburg, Chainability and Hemmingsen's theorem, Topology and its Applications 153 (2006), 2462-2468. (http: www.mscs.mu.edu/ paulb/Paper/chainable.pdf)

[3] P. Bankston, A survey of ultraproduct constructions in general topology, Topology Atlas Invited Contributions 8 (2003), 1-32. (http://at.yorku.ca/t/a/i/c/48.htm)

[4] - The Chang-Eoś-Suszko theorem in a topological setting, Archive for Mathematical Logic, 45 (2006), 97-112. (http:www.mscs.mu.edu/ paulb/Paper/topsetting.pdf)

[5] - On the first-order expressibility of lattice properties related to unicoherence in continua, Archive for Mathematical Logic (to appear). (http:www.mscs.mu.edu/ paulb/Paper/unicoh.pdf)

[6] _ Not every co-existential map is confluent, Houston J. Math. 36 (2010), 1233-1242. (http:www.mscs.mu.edu/ paulb/Paper/conf .pdf) 
[7] _ Defining topological properties via interactive mapping classes Topology Proceedings 34 (2009), 39-45. (http:www.mscs.mu.edu/ paulb/Paper/classm.pdf)

[8] C. C. Chang and H. J. Keisler, Model Theory, third ed., North Holland, Amsterdam, 1990.

[9] J.J. Charatonik, Unicoherence and multicoherence, in: Encyclopedia of General Topology (K. P. Hart, J. Nagata, J. E. Vaughan, eds.), Elsevier, 2004, pp. 331-333.

[10] R. Engelking, Outline of General Topology, North Holland, Amsterdam, 1968.

[11] K. P. Hart, A concrete co-existential map that is not confluent (preprint). (http://aw.twi.tudelft.nl/ hart)

[12] C. W. Henson, C. G. Jockusch, Jr., L. A. Rubel, G. Takeuti, First order topology, Diss. Math. 143 (1977), 1-40.

[13] H. J. Keisler, Model Theory for Infinitary Logic, North Holland, Amsterdam, 1971.

[14] J. Krasinkiewicz and P. Minc, Mappings onto indecomposable continua, Bulletin de l'Académie Polonaise des Sciences. Série des Sciences Mathématiques, Astronomiques et Physiques 25 (1977), 675-680.

[15] A. Lelek, On confluent mappings, Colloq. Math. 15 (1966), 223-233.

[16] W. Lewis, The pseudo-arc, Bol. Sci. Mat. Mexicana (3) 5 (1999), 25-77.

[17] S. B. Nadler, Jr., Continuum Theory, an Introduction, Marcel Dekker, New York, 1992.

[18] H. Wallman, Lattices and topological spaces, Ann. Math. (2) 39 (1938), 112-126.

[19] D. C. Wilson, Open mappings of the universal curve onto continuous curves, Trans. Amer. Math. Socl, 168 (1972), 497-515.

Department of Mathematics, Statistics and Computer Science, Marquette UniverSity, Milwaukee, WI 53201-1881, U.S.A.

E-mail address: paulb@mscs.mu.edu 